\section{Cahiers d'ethnomusicologie}

Anciennement Cahiers de musiques traditionnelles

12 | 1999

Noter la musique

\title{
Mali. Musique bambara du Baninko
}

Collection Archives Internationales de Musique Populaire (Musée d'ethnographie de Genève), en collaboration avec le Musée National du Mali, Bamako. 1998

\section{Vincent Zanetti}

\section{OpenEdition}

\section{Journals}

Édition électronique

URL : http://journals.openedition.org/ethnomusicologie/879

ISSN : 2235-7688

Éditeur

ADEM - Ateliers d'ethnomusicologie

Édition imprimée

Date de publication : 1 janvier 1999

Pagination : 240-242

ISBN : 978-2-8257-0680-0

ISSN : 1662-372X

Référence électronique

Vincent Zanetti, "Mali. Musique bambara du Baninko », Cahiers d'ethnomusicologie [En ligne], 12 | 1999,

mis en ligne le 08 janvier 2012, consulté le 01 mai 2019. URL : http://journals.openedition.org/ ethnomusicologie/879

Ce document a été généré automatiquement le 1 mai 2019.

Tous droits réservés 


\section{Mali. Musique bambara du Baninko}

Collection Archives Internationales de Musique Populaire (Musée d'ethnographie de Genève), en collaboration avec le Musée National du Mali, Bamako. 1998

\section{Vincent Zanetti}

\section{RÉFÉRENCE}

Mali. Musique bambara du Baninko. Enregistrements: Edda Brandes. Textes (en français et en anglais): Salia Malé, Edda Brandes et Josué Thierno. Collection Archives Internationales de Musique Populaire (Musée d'ethnographie de Genève), AIMP LVIII, en collaboration avec le Musée National du Mali, Bamako. 1 CD VDE 980, 1998

Dans le Mali contemporain, le groupe ethnique le plus important numériquement est celui que la littérature ethnographique a définitivement contribué à faire connaitre sous le nom de «Bambara». Installés sur un vaste territoire recouvrant grosso modo le quart sud-est du pays, les Bambara, qui dans leur propre langue se désignent en fait sous le nom de bamananw (au singulier: bamanan), se distinguent les uns les autres selon leur répartition géographique, dans les trois régions suivantes: Beledugu, Kaarta et Baninko.

Le présent disque s'est intéressé exclusivement aux musiques traditionnelles de cette dernière partie du pays bamanan, laquelle s'étend entre les cercles de Buguni et de Dioïla, au sud-est de Bamako. Il constitue la première concrétisation d'un projet conçu en 1989, dont le principal objectif était la constitution d'archives musicales détaillées sous forme de cassettes audio et vidéo. La grave crise politique - et forcément économique - qu'a traversée le Mali dans les années qui ont suivi cette heureuse initiative, en a bien évidemment ralenti l'exécution. Cela ne donne que plus de valeur à ces enregistrements, lesquels participent dès lors à leur manière à l'image d'un Mali nouveau, soucieux de se montrer dans toute sa diversité culturelle et de faire oublier les graves conflits ethniques qui ont secoué sa partie saharienne. Pour les réaliser, six chercheurs ont visité vingt-deux villages du Baninko, entre 1991 et 1992, invitant à chaque fois les habitants à présenter une spécialité musicale qui les distingue des villages voisins. D'où une heureuse diversité 
des pièces, particulièrement intéressante, pour ne pas dire rafraîchissante, en des temps où le public occidental a trop souvent tendance à assimiler le patrimoine musical traditionnel d'Afrique de l'ouest avec les seules percussions, et en particulier avec le tambour jembe. Ordonnées en une succession attrayante, les pièces permettent de se familiariser avec des instruments à cordes (luth bamanan'goni à quatre cordes des griots de caste, harpe-luth à chevalet donzon'goni des soraw, les chantres des chasseurs), avec des trompes, des sifflets, des percussions très variées, mélodiques ou purement rythmiques, et bien sûr, toute une gamme de genres vocaux différents, tant masculins que féminins.

3 La qualité des prestations musicales est toujours très bonne et c'est un vrai plaisir de constater que certaines traditions, pas forcément reconnues dans le Mali moderne des villes, parviennent encore à survivre dans le monde rural. Ca n'est malheureusement pas toujours le cas et on a bien souvent entendu des personnes d'âge mûr, notamment des gens originaires du Baninko, se plaindre que leurs villages n'étaient plus animés comme par le passé et que les jeunes gens n'étaient plus motivés à entretenir leur patrimoine culturel.

4 Cela dit, les enregistrements ne sont pas toujours à la hauteur des manifestations qui en font l'objet: une part importante de la matière musicale ne parvient malheureusement pas jusqu'à l'auditeur, ou alors d'une façon bien déformée. Ainsi le chant du griot Fotigui Jabaté et le timbre de son n'goni perdent beaucoup de leur profondeur, parce qu'enregistrés de trop loin dans une pièce trop réverbérante, alors qu'on aurait certainement pu l'inviter à jouer à l'extérieur et lui consacrer deux microphones séparés. Même constat, ou presque, pour Abel Malé et son donzon'goni, dont la chaleur et la profondeur des notes basses ne sont pas du tout perceptibles ici. De même encore pour les chœurs de femmes qui sont censés faire naître le rythme et la danse Bonjalaa, mais qui sont ici complètement couverts par le jeu des tambours. C'est d'autant plus malheureux que tant que les ethnomusicologues ne seront pas plus attentifs à la qualité de leurs prises de son, ils ne toucheront jamais qu'un public restreint d'érudits et de spécialistes. A une époque où le marché de la world music tend de plus en plus à influencer les répertoires traditionnels en fonction de la demande artistique des scènes occidentales, les traditionalistes sont en droit de se sentir trahis: c'est leur image qui est en jeu et c'est bel et bien eux que l'on juge, consciemment ou non, à travers ces enregistrements.

5 Ceci dit, il y a tout de même des plages très fortes, comme celles consacrées à l'orchestre de trompes buru, enregistrées dans le village de Balun. Et puis il y a le texte de Salia Malé, très riche et pertinent, qui parvient en quelques pages à rendre accessibles la conception bamanan de la musique et sa relation indissociable avec la parole chantée et avec la danse: c'est le foli, «la manifestation culturelle où la modulation du chant, le rythme de la musique et l'exécution de figures de danse concourent pour donner à l'expression son plein sens de dire.»

6 En conclusion, de par sa diversité, de par la richesse de sa présentation et bien sûr, de par la qualité des interprètes, ce disque des musiques bamanan du Baninko parvient à rester une œuvre attachante, malgré ses défauts de réalisation. Il représente un témoignage indispensable de la survie d'une certaine manière de penser et de vivre la musique et rappelle la richesse de la culture bambara traditionnelle. On ressort de son écoute en attendant la suite, ce qui est déjà une marque de qualité. 